\title{
A critical narrative review of radiotherapy for retroperitoneal soft tissue sarcoma
}

\author{
Clara Le Fevre, Waisse Waissi, Isabelle Chambrelant, Georges Noel^, Delphine Antoni \\ Department of Radiation Oncology, Institut de Cancérologie Strasbourg Europe (ICANS), 17 Rue Albert Calmette, BP 23025, 67033 Strasbourg, \\ France \\ Contributions: (I) Conception and design: G Noel, W Waissi; (II) Administrative support: G Noel; (III) Provision of study materials or patients: C Le \\ Fevre, G Noel, W Waissi, D Antoni; (IV) Collection and assembly of data: C Le Fevre, W Waissi, G Noel; (V) Data analysis and interpretation: C \\ Le Fevre, G Noel; (VI) Manuscript writing: All authors; (VII) Final approval of manuscript: All authors. \\ Correspondence to: Prof. Georges Noel, MD, PhD. Department of Radiation Oncology, Institut de Cancérologie Strasbourg Europe (ICANS), 17 Rue \\ Albert Calmette, BP 23025, 67033 Strasbourg, France. Email: g.noel@icans.eu.
}

\begin{abstract}
Retroperitoneal sarcomas (RPSs) are rare diseases, and the 5-year overall survival rate remains low. Management of RPSs is challenging, and the quality of treatment strategy is a crucial prognostic factor. Treatment decisions and the treatment department to which the patients is referred must be centralized. Overall survival rate is positively correlated with the number of patients treated in the centers. En bloc surgery is the standard of care for effective treatment and potential for cure, whereas perioperative chemotherapy or radiotherapy has not yet been validated. Pre- or postoperative radiotherapy was considered controversial, but the STRASS trial publication illustrated that preoperative radiation could be useful in some conditions. Retroperitoneal liposarcoma might become a future target of prospective trials. Furthermore, some retrospective studies have shown that preoperative radiotherapy increases the rate of R0-R1 resections. This article reviews the role of external beam radiotherapy in the management of RPS and highlights the optimal volumes, doses, and radiotherapy techniques for the treatment of RPS. As accurately predicting prognosis in patients with RPS is challenging, we also emphasize the utility of nomograms in the field of radiotherapy. However, these nomograms do not include radiotherapy as a predictive factor. Although some authors could conclude that use of radiotherapy should be avoided based on the results of the STRASS trial, some data argue that radiotherapy still has utility in select cases.
\end{abstract}

Keywords: Retroperitoneal sarcoma (RPS); radiotherapy; intensity-modulated radiotherapy (IMRT); nomogram; review

Submitted Aug 22, 2020. Accepted for publication Nov 17, 2020.

doi: $10.21037 / \mathrm{cco}-20-209$

View this article at: http://dx.doi.org/10.21037/cco-20-209

\section{Introduction}

Soft tissue sarcomas (STSs) are rare and heterogeneous malignancies. In a prospective population-based study performed between March 2005 and February 2007, Ducimetière et al. reported a crude incidence rate of 6.4 cases per 100,000 , representing $1 \%$ of all new yearly cancers (1). Approximately, 50\% of STSs occur in the limbs, while $9 \%$ to $15 \%$ occur in the retroperitoneum (2). The recently updated World Health Organization (WHO) classification of soft tissue and bone tumors recognizes 113 histologic subtypes of STSs that are classified into 12 categories (3). The most common subtypes include liposarcomas (LPSs), undifferentiated-unclassified tumors,

$\wedge$ ORCID: 0000-0003-4952-5572. 
and leiomyosarcomas. Over the last decades, several specific genetic alterations have been described, allowing a molecular classification $(4,5)$.

Surgery is the corner-stone of RPS treatment, but complete resection is often difficult or impossible due to the large tumor size and involvement of adjacent vital structures. Based on an extensive series in the literature, resections with negative margins are challenging to achieve and gross tumor resection, including $\mathrm{R} 1$ resection, are in $54 \%$ and $67 \%$ of cases (6). Consequently, in multidisciplinary treatment meetings, neoadjuvant and adjuvant treatment are regularly disputable proposals. Radiotherapy is the most frequent option, but its use always remains controversial. This article reviews the literature with a critical mind for the goal of showing the advantages of care conveyed by a well-conducted radiation therapy in the context of expert global management. We present the following article in accordance with the Narrative Review reporting checklist (available at http://dx.doi.org/10.21037/ cco-20-209).

\section{Initial evaluation}

\section{Specific evolution of sarcomas}

Retroperitoneal sarcomas (RPS) are often diagnosed late because they tend to be painless enlarging masses (7). In a recent sizeable multi-institutional review, the median size at diagnosis was reportedly $20 \mathrm{~cm}$ (8). Approximately $40 \%$ of RPSs are low grade, while the remaining $60 \%$ are intermediate or high-grade (9). Low-grade tumors infrequently metastasize, while intermediate and high-grade tumors can metastasize primarily to the lung and liver (10). Despite these initial sizes and grade, the incidence of distant metastasis at diagnosis is low, approximately $10 \%$, occurring in a minority of patients $(11,12)$. Specifically, for RPS, the most common site of local recurrence is in the retroperitoneum. This kind of evolution pattern in favor of local complementary treatment rather than systemic chemotherapy or targeted treatment.

\section{Imaging workup}

Because multimodal imaging improves tumor delineation and consequently intra- and interradiation oncologist delineations, the more relevant imaging and sequencing must be performed to consistently strengthen and homogenize delineation. For RPS diagnosis, a computed tomography (CT) scan of the abdomen and pelvis is usually performed, followed by a core needle biopsy. LPSs have some specific radiological features. Well-differentiated LPSs have typically large areas of abnormal fat, while higher density nodules are usually seen in dedifferentiated LPSs. Using CT scans, Lahat et al. accurately identified $100 \%$ of the well-differentiated LPSs (13). In contrast, leiomyosarcomas typically appear as heterogeneous, solid tumors. MRI scans help delineate the treatment target, particularly for pelvic tumors, but are less commonly performed for lesions above the pelvic brim.

Nevertheless, MRI is generally preferred over CT scan for both diagnostic characterization and staging purposes for planning effective management (14). Indeed, T1weighted images provide an excellent anatomic definition and are typically relied upon for preoperative planning. As the sequence is not fluid sensitive, T2-weighted sequences evaluate the edema. Specific MRI sequences such as short tau inversion recovery (STIR) or fat saturation (FAT-SAT) are useful for the evaluation of lipomatous tumors (15). The delineation between tumor tissue and healthy tissue is improved by T1-weighted contrastenhanced with gadolinium MRI use (16).

PET scan technology can assess the in vivo tumor metabolic activity via positron-emitting radionuclides. Fluorodeoxyglucose-18 $\left({ }^{18} \mathrm{~F}-\mathrm{FDG}\right)$ is the most commonly used radionuclide. In a recent publication, diagnostic and prognostic of PET/CT in sarcoma were considered highly sensitive and specific in the detection of STS (17) and RPS (18). Furthermore, the SUVmax of the primary tumor was a strong predictor of survival (19). Although FDG-PET has demonstrated potential benefits, the use of FDG-PET in STS is still considered investigational.

Because of the risk for metastases, at least in patients with intermediate and high-grade tumors, a CT scan of the chest should be performed to evaluate for pulmonary metastases before the decision to administer irradiation.

Although the useful of MRI and FDG-PET for diagnosis is not disputable, their utility for delineation remains weak because of the motion of abdominal cavity secondary to the breath or to digestive peristalsis. Moreover, the heterogeneity of this tumor after gadolinium injection or radio-isotope uptake alter the quality of delineation. Some digestive artifacts after FDG uptake can mimic a tumor location or a residual tumor after surgical removal. Furthermore, with FDG-PET, inflammatory reactions can interfere with the interpretation of postoperative images. 


\section{Biopsy}

When a sarcoma is suspected, the biopsy should be performed with care and consideration of the definitive resection procedure. Optimally, the biopsy is performed by the same surgeon who will perform the definitive resection (2). Core needle biopsy is minimally invasive and more accurate than fine-needle aspiration (20). If an incisional biopsy is required, it should be performed in line with the skin incision of the planned resection of the malignant lesion. In a published series of 177 retroperitoneal LPSs, 56\% were well-differentiated, 37\% were dedifferentiated, and $7 \%$ were myxoid-round cell subtypes $(21,22)$. Approximately $40 \%$ of RPSs are low grade, while the remaining $60 \%$ are intermediate or highgrade (9).

\section{Management in a multidisciplinary bigh-volume center}

Because RPS is a rare and complex disease, patients with RPS should be managed in expert high-volume multidisciplinary sarcoma centers. There is emerging evidence that outcomes are improved when physicians with expertise treat patients compared to those who were not. Gutierrez et al. published an analysis of patients treated for RPS in the Florida Cancer Data System. They concluded that OS was improved in patients treated at a high-volume center (23), which was subsequently confirmed by several additional publications (8,24-27). One of the key reasons that could explain such a difference is the surgeons' experience. Indeed, the role of surgical expertise is an essential part of preserving tumor integrity during surgery, and many studies have demonstrated that ruptured tumors are a negative prognostic factor of overall survival. Data from the French Association of Surgery showed a correlation between a high caseload per center and abdominal recurrence-free survival (24). This result is partly attributed to the higher number of radical resections performed but also to the lower tumor rupture rates seen in high-volume centers. The significance of microscopic margins is unclear, although there is some evidence for better outcomes with R0 than with R1 resection (28). Marginal or incomplete (R1 or R2) resection has been reported in up to $50 \%$ of the patients treated with curative intent. In these patients, local relapse appeared in $2 / 3$ of them even though local failure rose to half of the patient after complete resection (29). These observations suggest neoadjuvant or adjuvant treatments to improve outcomes.
In the postoperative incomplete resection situation, the preoperative implementation of metallic clips by the surgeon in the R1 areas dramatically helps the radiationoncologist to delineate these locations and to prescribe increased doses. However, for RPS, R0 and R1, resection could be acceptable but R2 should be avoided as much as possible. Indeed, in Gronchi's sarculator nomogram, R0 and R1 were regarded the same, with no distinction (30). However, an adjuvant radiotherapy (AdjRT) should be considered as an exception when a neoadjuvant radiotherapy (neoRT) cannot be performed, but for these rare cases, when an R1resection is suspected by the surgeon, the R1 area should be marked using clips.

Notably, the determination of histological diagnosis and prognostic factors is essential for defining treatment strategies. Ray-Coquard et al. showed in the analysis of three European region databases that more than $40 \%$ of histological diagnoses were modified after the second reading in a specialized center (31).

Chemotherapy is minimally effective in most of the cases. Nevertheless, it may play a role in downsizing borderline resectable sarcomas and treating some chemosensitive RPSs as such as leiomyosarcoma, undifferentiated pleomorphic sarcoma, and dedifferentiated LPSs (32-34). As much as possible, patients should be included in trials because efficient treatments are being evaluated, and phase I or II studies are regularly proposed. These trials require organizational structure usually reserved for academic hospitals because of their level of expertise. Expert centers can also improve the efficacy of recruitment into trials because of the regular patient volume $(35,36)$.

Radiotherapy can propose a large panel of techniques to irradiate these complex volumes. Departments with a great experience with various modern machines and, with optimized planning strategies are preferred for optimal and safe irradiation.

\section{External beam radiotherapy treatment modality}

To improve local control, different schedules of radiotherapy have been developed [preoperative (NeoRT), intraoperative (IORT), and postoperative (AdjRT)] (37). In contrast to extremity STSs, there has been no randomized trial evaluating the benefit of radiotherapy for RPS. The American College of Surgeons Oncology Group phase III trial (ACOSOG Z9031) was launched to determine the effect of adding preoperative RT for RPS treatment. The trial closed prematurely due to a lack of patient accrual. 
The NCCN guidelines, as well as the consensus statement by the Trans-Atlantic RPS Working Group recommend consideration of NeoRT in select patients but do not support the use of AdjRT, except in isolated cases where it is deemed necessary $(38,39)$. More recently, the EORTCSTBSG 62092-22092 trial (STRASS) is randomizing patients between surgery with or without NeoRT. This trial recruited over 6 years and closed after completing the accrual. However, the results are not as definitive as they seem (40).

\section{Radiotherapy schedules}

Radiotherapy to the retroperitoneum is quite complicated procedure whatever the schedule. With the newest RT techniques, such as IMRT, the surrounding healthy tissues can be shielded, and acute radiation-induced adverse events rates were dramatically decreased (41).

IORT is an elegant method to deliver doses into a suspicious $\mathrm{R} 1$ area. This schedule requires a high dose, delivered in one fraction, always close to healthy, radiosensitive tissues. Consequently, doses and irradiated volumes remained limited, and this schedule is always combined with AdjRT $(42,43)$.

In a recent review discussing surgery in RTS, Peacock et al. noted that NeoRT was more frequently used in academic hospitals, although AdjRT was preferred in local hospitals. Furthermore, NeoRT was more frequent during the 2007-2011 period than during the 2003-2006 period (29). Two positive factors of outcome improvement, academic hospital and recent period, favoring the use of NeoRT, can be considered biases. Indeed, academic hospitals can near the number of managed patients, and the recent period can be parallelized with the newest techniques of radiotherapy allowing to a decreased of radio-induced side-effects, which is specifically useful in NeoRT.

Nussbaum et al. queried the US National Cancer Database (NCDB) for all RPSs treated with NeoRT or AdjRT or surgery alone. Two case-control propensity score-matched datasets were created, one for patients who received NeoRT $(\mathrm{N}=563)$ compared to those who had surgery alone $(\mathrm{N}=1,126)$ and another for patients who received AdjRT $(\mathrm{N}=2,196)$ compared to those who had surgery alone $(\mathrm{N}=2,196)$. In this largest propensity scorematched study, the authors showed that both NeoRT (HR: 0.70, 95\% CI: 1.01-2.36, P<0.0001) and AdjRT (HR: 0.78; 95\% CI: $1.01-2.36, \mathrm{P}<0.0001)$ improved OS compared to surgery alone (43). However, Chouliaras et al. performed equivalent propensity analysis of 46 patients treated with NeoRT compared to 46 nonirradiated patients, and 59 patients treated with AdjRT compared to 59 nonirradiated patients. In both groups, there were no significant differences in terms of LR, DFS, or OS (42); the disparity in number of patients between the two studies could explain the differences in the results.

A recent meta-analysis could be considered optimistic because, among 3,958 patients, authors concluded that OS and LR were statistically improved with external beam radiotherapy in patients with RPS. NeoRT seemed more efficient in LR than AdjRT but with increased wound complications. However, the results remained disputable due to the heterogeneity of the patient presentations and radiotherapy techniques (44). In 2020, a new metanalysis analyzed the perioperative irradiation. The authors showed that NeoRT and AdjRT improved OS and 5-year OS compared to surgery alone [weighted mean difference (WMD): $-22.93 ; 95 \%$ CI: $-27.91,-17.96 ; \mathrm{P}<0.0001]$ and (WMD: -18.93 ; 95\% CI: $-19.13,-18.74 ; \mathrm{P}<0.0001$ ), comparatively. Median RFS was also significantly increased in patients treated with either surgical resection and NeoRT or surgical resection (WMD: 0.19; 95\% CI: 0.11, 0.33; $\mathrm{P}<0.0001$ ) and AdjRT (WMD: 0.49; 95\% CI: 0.32, 0.75; $\mathrm{P}=0.001$ ) compared to surgery alone (45).

The use of NeoRT is advantageous for several reasons, proven or theoretical. Radiotherapy downstages the tumor, enables more limited surgery, and reduces the number of healthy tissues that require removal. Furthermore, in some cases, radiotherapy allows complete removal of initial marginally resectable tumors. Radiotherapy may increase surgical margin negative resection $(46,47)$ and peroperative tumor cell seeding. Compared to AdjRT, NeoRT improves response because the tumor in place is more radiosensitive due to improved oxygenation. Furthermore, after surgery, in the tumor bed, the healthy tissue fills the operative cavity leading to irradiation of more healthy tissues.

\section{Target volume and organ at risk delineations}

\section{Simulation}

Before CT acquisition, patients should be placed in the supine position with arms resting in supports over the head and should be immobilized with vacuum cushions. To enhance target delineation and help identify the gastrointestinal tract, intravenous contrast injection or oral contrast ingestion can be performed (48). Slice thickness 
acquisition should be $<3 \mathrm{~mm}$ according to a recent international guideline (49). Authors have demonstrated that tumors and organs located in the upper abdomen may move significantly due to respiration (50). For this reason, 4-dimensional CT scan acquisition is mandatory for tumors above the iliac crest (49). Consequently, gating or abdominal compression devices can be used to limit breathing motion. Registration of post gadolinium T1weighted magnetic resonance imaging (MRI) may assist target volume delineation (51). MRI performed using a flat couch and with the patient in the treatment position are mandatory to usefully match CT scan and MRI.

\section{Delineation of organs at risk (OARs)}

The following OARs should be delineated: spinal cord, stomach, intestinal cavity (bowel bag), rectum, liver, kidneys, bladder, uterus, ovaries or testis, and femoral heads. Regarding intestinal cavity delineation, sarcoma experts have contoured small bowel and colon as the bowel bag. This latter approach has multiple advantages. First, it is easier and faster to contour compared to each small bowel loop. Moreover, as the small bowel is an organ with a large amount of movement, delineating each bowel segment overestimates the small bowel receiving more than 45 Gy (52). Thus, the EORTC trial (STRASS) recommended delineating the bowel bag as a structure containing all intestinal segments. The other OARs should be contoured according to existing guidelines (53). Furthermore, due to the volume of some sarcomas, the digestive tract can be compressed and moved, making it difficult to differentiate between small bowel loop and colon, so justifying the use of a bowel bag delineation.

\section{Delineation of target volumes}

Accurate delineation of radiation target volumes is a prerequisite for the precise delivery of the radiation dose to the region of interest. This delineation is currently the central issue in the era of image-guided intensity-modulated radiotherapy (IG-IMRT).

Different high-volume sarcoma centers have published their contouring approaches and showed differences (ranging from 5 to $20 \mathrm{~mm}$ ) between the margins from GTV to CTV among those centers (47,54-56). Only one publication from the MD Anderson Cancer Center group described a margin of $45 \mathrm{~mm}$ due to volume motion during respiration (57). Therefore, as mentioned above, the importance of using 4D-CT scans for delineation and devices to limit this motion are pivotal approaches fir reducing the internal margins (50).

Currently, the standard guidelines for RPS delineation are based on the NSABP, RTOG, GOG (NRG) consensus agreement among experts (48). Twelve international experts contoured two cases of RPS, and the agreement was "nearly perfect" according to a kappa value between 0.84 and 0.92 . CTV was defined as a GTV expansion of $15 \mathrm{~mm}$ with edited reduction at the bone, bowel bag and air cavity $(5 \mathrm{~mm})$, renal and hepatic interface $(2 \mathrm{~mm})$, and skin surface $(3 \mathrm{~mm})$. When a 4D-CT scan was obtained, the authors specified that the reduction to the renal and hepatic interface was $0 \mathrm{~mm}$ because the 4D-CT scan considers organ motion due to respiration (50). However, GTV should be delineated on the maximum intensity projection (MIP) sequence and healthy tissues on the average sequence.

After NeoRT, there is still a risk of retaining a positive margin after resection. Therefore, the authors defined "high-risk CTV" (HR-CTV) as the area to be at high risk of a positive margin after resection. The HR-CTV should be contoured with the aid of surgeons, as it includes organs that surgeons would leave in situ as along with the posterior wall, para- and prevertebral space, and major vessels. In contrast to GTV and CTV, the HR-CTV delineation agreement among the panel of experts was quite moderate (Kappa values of 0.50 and 0.57 ) (58).

There is no consensus guideline for the definition of the planned target volume (PTV). The STRASS trial recommends a $9 \mathrm{~mm}$ expansion in the anterior and medial directions, whereas a $12 \mathrm{~mm}$ expansion is recommended in other directions $(59,60)$. However, daily IGRT could help reduce range uncertainties, decreasing the PTV margin. In a series of RPS patients treated with IG-IMRT, authors from Princess Margaret Hospital evaluated temporal and volumetric changes in RPS with weekly cone-beam CT. They found that after a slight volume increase, the GTV volume decreased from the beginning to the end of radiotherapy. Moreover, they showed GTV interfraction displacement of $15 \mathrm{~mm}$ in the superior/inferior and anterior/posterior directions and $8.6 \mathrm{~mm}$ in the lateral direction. The authors emphasized the benefit of adaptive radiotherapy, allowing replanning when necessary (50).

\section{Prescription dose}

Some studies have shown a relationship between delivered doses and patient outcomes (61). In the STRASS trial, the 
prescription dose was 50-50.4 Gy in 1.8-2 Gy fractions, and at least $95 \%$ of the PTV should receive $95 \%$ of the prescription dose (60). As mentioned above, there has been an interest in the concept of dose escalation to a volume of the high-risk of positive margins (47,61-63). Dose escalation is feasible with recent techniques of radiotherapy, such as IMRT or proton therapy. When radiotherapy is performed postoperatively, dose escalation is limited by late gastrointestinal (GI) toxicity. Several publications observed a significant incidence of late GI complications between 50 and 60 Gy $(61,64-66)$.

\section{Irradiation technique}

Since it was introduced for the treatment of RPS, photonbased RT has evolved from 3-dimensional conformal radiotherapy (3D-CRT) to IMRT and image-guided radiotherapy (IGRT). The goal of these modalities is to improve outcomes while minimizing acute and long-term complications. Indeed, IMRT is a conformal technique that improves target coverage while reducing the dose to neighboring at risk organs. Many authors have reported the dosimetric superiority of IMRT compared to 3D-CRT in treating RPS patients $(56,67,68)$. Another advantage of IMRT is that it allows dose escalation to areas of gross disease or in the R1 area in the case of AdjRT or in HR-CTV in case of NeoRT. Indeed, authors from the University of Alabama published their experience using IMRT for NeoRT to treat RPS. They were able to deliver both 45 Gy in 25 fractions of 1.8 Gy to the entire GTV and a simultaneous integrated boost (SIB), allowing a total dose of 57.5 Gy in 25 fractions of 2.3 Gy to the posterior retroperitoneal surgical margin at high risk for recurrence. With an actuarial two-year local control rate of $80 \%$, one patient experienced radiation-related grade 2 late toxicity, and treatment morbidity was acceptable for the remaining patients (47). Another approach from a group at Leuven, Belgium, tested NeoRT in 83 patients, targeting the area of contact between the tumor and the posterior abdominal wall. They delivered a total dose of $50 \mathrm{~Gy}$ in 25 fractions of 2 Gy per day with IMRT or conformal radiotherapy. They reported low toxicity rates with 6 cases of CTC grade 1-2 side effects and only two patients with CTC grade 3 anorexia. All patients except one had complete surgical excision (62). Sargos et al. published data from the TOMOREP phase II trial evaluating the efficacy and toxicity of 54 Gy with helical tomotherapy for NeoRT to treat retroperitoneal LPS and showed that it was feasible and mostly well tolerated (69).

Particle therapy, such as proton therapy or carbon ions seems to be promising. Proton therapy is currently the most widely used form of the charged particles. Because of the absence of an exit dose beyond the Bragg peak, protons can spare adjacent organs, allowing dose escalation (70). Because radiosensitive organs such as the small bowel or kidneys are often close to the target volumes, patients with RPS may benefit from the use of charged particles. Compared to the evolution of photon-based irradiation from 3D-CRT to IMRT, delivery of protons evolved from passive scattering to pencil beam scanning, offering improved treatment conformity (70). DeLaney et al. showed in a recent phase 1 publication that proton therapy might be an excellent tool for further dose escalation into the high-risk of relapse volume (63). Patients were treated with 50.4 Gy $\mathrm{G}_{\mathrm{RBE}} \mathrm{NeoRT}$ with intensity-modulated proton therapy (IMPT) with simultaneous integrated boost dose escalation from 60.2 to $63 \mathrm{~Gy}_{\mathrm{RBE}}$. They showed that dose escalation to $63 \mathrm{~Gy}_{\mathrm{RBE}}$ was feasible without acute dose-limiting toxicity. Compared to protons, carbon ions have comparable physical dose distributions and higher relative biologic effects. A group in Chiba, Japan, studied dose escalation with carbon ion radiotherapy in 24 patients with unresectable RPS. The mean dose was 68.9 GyE (range, 52.8-73.6 GyE). At five years, local control and overall survival rates were $69 \%$ and $50 \%$, respectively. No patients experienced GI toxicity, but $21 \%$ of patients experienced long-term neurological complications (71).

Irrespective of the IMRT technique or proton therapy, IGRT is frequently performed just before radiation therapy treatment sessions to verify the target position and enable the safe use of conformal high-dose irradiation (69). Adaptive radiotherapy is an approach to correct for morphological changes in the patient's anatomy, such as tumor and normal tissue variations as a result of treatment. Wong et al. showed that tumor volume during radiotherapy could change, and replanning could be mandatory when needed (50). Comparably, among 23 patients treated for STS, authors showed that growth of the tumor was observed six times requiring a new delineation in four patients (72). This drawback of the NeoRT was also reported in the STRASS trial in $16 \%$ of patients. These failures did not change the general conclusion of the trial but, in absence of an analysis, enlarged the difference of relapse between both arms in favor of neoRT (60). 


\section{Outcomes}

In previously published series, outcomes depended on a large variety of factors, including histologic grade, tumor extent, adjuvant therapy, and the proportion of patients treated for recurrent disease. Furthermore, the expertise of the surgical team likely influences prognosis. In general, there was a clear trend toward using NeoRT to reduce the local recurrence rate (73). However, given the historical difficulty of enrolling patients with RPS in randomized trials, no benefit has been observed in OS, and when expected recruitment was completely reached, the results remained disappointing (35). This lack of OS improvement may be surprising, as in RPS patients, mortality occurs secondary to local relapse $(11,22,74)$.

The main problem with this kind of analysis is the multiple biases gathered in the same series. Among 400 patients, Chouliaras et al. obtained groups with no radiotherapy, NeoRT or AdjRT both combined with chemotherapy, or not. Even with propensity scoring results were not convincing and the authors concluded an absence of difference in OS, LR, or DFS, regardless of NeoRT or an AdjRT combination or schedule (42). These results are in disagreement with studies recently published, including a recent meta-analysis that found improved LR rates in response to NeoRT and AdjRT $(12,44,75)$. The heterogeneity in histologic subtypes, tumor grade, and resection status constitute well known potential sources for variability in the responses to radiotherapy. Toulmonde $e t$ al. analyzed a cohort of 586 patients from the French Cancer Center Federation Sarcoma Group, most of whom received multimodal treatment. Radiotherapy was delivered in 146 patients (29\%), primarily with an AdjRT schedule (74\%). In multivariate analysis, radiotherapy was favorably associated with local control (HR $=0.5$; 95\% CI: $0.4-0.7 ; \mathrm{P}<0.001)$. In a subgroup analysis, the authors showed that patients with dedifferentiated LPS exhibited better local control when treated with irradiation compared to surgery alone (HR $=0.6 ; 95 \%$ CI: 0.4-0.9; $\mathrm{P}=0.028)$ (75).

In 1993, Sindelar et al. reported a randomized prospective trial comparing AdjRT (50-55 Gy) and IORT (20 Gy) plus AdjRT (35-40 Gy) in 35 patients treated for RPS (76). With a median follow-up of 8 years, add IORT before EBRT decreased local recurrence compared to EBRT alone $(40 \%$ vs. $80 \%)$. However, the authors did not show a difference in OS and demonstrated substantial toxicity (50-60\%) in both arms. Although the authors showed a benefit in local control, EBRT+/- IORT was not widely adopted due to of the toxicity and the lack of a survival benefit. Furthermore, treating sarcoma patients with IORT is more challenging, both technically and from an organizational point of view. Recently, Kirste et al. reported a combination of NeoRT, IORT, and AdjRT with an acceptable tolerance (77). However, this approach has to be evaluated in lerger populations before becoming routine.

Many retrospective studies have evaluated the role of EBRT in decreasing local recurrence.

Gieschen et al. published on 37 RPS patients who received 45-50.4 Gy NeoRT and showed that complete resection was achieved in $78 \%$ of cases (78). Recently, the results of the STRASS trial were presented and failed to demonstrate the advantages of NeoRT. Patients were randomized between NeoRT, followed by surgery or surgery alone. A preponderance of patients $(74.5 \%)$ had LPS. The primarily endpoint was abdominal recurrencefree survival (ARFS). For overall and LPS patients, 3-year ARFS was $60.4 \%$ (95\% CI: 51.4-68.2\%) and 58.7\% (95\% CI: 49.5-66.7\%) $(\mathrm{P}=0.954)$ and $71.6 \%(95 \% \mathrm{CI}: 61.3-$ $79.6 \%)$ and $60.4 \%(95 \% \mathrm{CI}: 49.8-69.5 \%)(\mathrm{P}=0.049)$ in NeoRT versus surgery groups, respectively (35).

In 2001, Stoeckle et al. reported on a cohort of patients with nonmetastatic RPS from the French Cancer Center Federation Sarcoma Group (11). In that study, 94 patients $(65 \%)$ underwent complete resection, and 89 patients (61\%) received a median AdjRT dose of 50 Gy. For patients who underwent complete excision, the risk of developing local recurrence was decreased by 3.4-fold in patients who received AdjRT compared to patients who did not receive AdjRT. Furthermore, the 5 -year LC rates were $55 \%$ and $23 \%$, respectively $(\mathrm{P}=0.002)$. Multivariate analysis of local control revealed that a lack AdjRT $(\mathrm{P}=0.0002)$ and Grade 3 histology $(\mathrm{P}=0.0047)$ significantly decreased the probability of LC. In 2010, Sampath et al. reported results of 261 who underwent surgery; among them, 73 received perioperative radiotherapy. The local failure-free survival rate at 5 years was $69 \%$. AdjRT significantly improved LC compared to no radiation treatment $(\mathrm{HR}=0.42,95 \% \mathrm{CI}$ : $0.21-0.86, \mathrm{P}<0.05)$, with a 5 -year rates of $79 \%$ and $64 \%$, respectively (79). More recently, in one of the largest series ever reported of surgically managed RPS patients, Turner et al. analyzed 102 patients who underwent surgery for an RPS. NeoRT was delivered in 62 patients, and 40 (70\%) underwent surgery. Radiotherapy was associated with more frequent $\mathrm{R} 0$ resection $72.5 \%$ vs. $30.6 \%, \mathrm{P}<0.001$ ), but with multivisceral resection $(87.5 \%$ vs. $66.1 \%, \mathrm{P}=0.02)$. 
Median local relapse-free survival was superior in the group treated with NeoRT than in the group that received only surgery (89.3 vs. 28.4 months; $\mathrm{P}=0.04$ ). Similar results were observed for OS (119.4 vs. 75.9 months, $\mathrm{P}=0.04)$ (80). Some comparable results were also obtained in two previous series $(81,82)$. Snow et al. concluded that lack of NeoRT was a poor prognostic factor of LC (82). In a recent systematic review of NeoRT in RPS, Cheng et al. reported the results of 15 articles with 464 patients. They showed that patients treated with NeoRT experienced a median 5-year LC rate and OS of $75 \%$ and $58 \%$, respectively (83). As a comparison, a median OS of 33 to 49 months was reported among patients who underwent surgery alone, and the recurrence-free survival rate was only $23 \%$ (37).

The Scandinavian Sarcoma Group analyzed a cohort of 97 patients. Forty-two patients received radiotherapy (88\% AdjRT). RT was significantly associated with improved LC, resulting in a 5 -year LRFS of $77 \%$ compared to $39 \%$ without RT ( $\mathrm{P}=0.001)$. Furthermore, the 5 -year OS was $71 \%$ in the RT group compared to $52 \%$ in the group that received surgery alone $(\mathrm{P}=0.019)$ (84). Recently, two studies were published using propensity score-matched cohorts to control biases inherent in the use of perioperative irradiation. Ecker et al. queried the NCDB for patients treated for retroperitoneal LPS by either surgery alone or NeoRT plus surgery. After identification of the covariables associated with OS, using the Cox regression model, the authors matched 173 patients treated with surgery alone and 174 patients treated with NeoRT by propensity scores and showed an improvement in OS (HR $=1.54 ; 95 \%$ CI: $1.01-2.36, \mathrm{P}=0.046)(85)$.

Large retrospectives studies can be informative. The Surveillance, Epidemiology, and End Results (SEER) database was recently used to identify patients with pathology confirmed RPS from 1973 to 2010. Among 480 patients, 144 received AdjRT. Patients who received AdjRT exhibited improved median OS compared to those who did not (36 vs. 27 months, $\mathrm{HR}=0.79, \mathrm{P}=0.023$ ) (86). Recently, in a report of the Trans-Atlantic Retroperitoneal Sarcoma Working Group (TARSWG) from 8 institutions and approximately 607 patients with RPS restricted to LPSs (stratified according to grade), perioperative radiation therapy was associated with improved local control in all cohorts. However, this association disappeared after controlling for confounders was introduced, and ultimately, no effect of radiation therapy on distant metastasis or OS was observed (40).

There are several possible reasons for these conflicting results. Sample size is an issue, given that all retrospective studies include a relatively small number of patients except large multicenter series but with less useful information. Treatment regimens are not always uniform within study groups, and many studies include patients with either NeoRT or AdjRT and sometimes IORT in a single treatment arm. Finally, there are arguments for the use of radiotherapy to prevent local relapse. Compared to AdjRT, the NeoRT approach could be more effective. However, except for a few of these studies, all failed to demonstrate a relationship between the gains in LC and OS. An analysis of treatment at relapse could be informative because reluctance to reoperate after previous RT could lead to a chance loss for the patients who received NeoRT or AdjRT at time of the first part of their disease. Consequently, postponing relapse is likely insufficient to improve survival. However, the issue of quality of life (QoL) was rarely assessed except in a publication of Wong et al. who focused on this topic (87). From 48 patients treated with NeoRT and surgery, QoL was correlated with complications. However, if these complications were correctly managed, QoL at 36 months was better than before treatment (87).

\section{Palliative radiotherapy}

When surgery is not an available option, radiotherapy can be an option to decrease symptoms and pain. Kepka et al. reported a large series of patients with various sarcomas. Patients with RPS represented $25 \%$ of the populiation. The OS was correlated with the size of the tumor. Complication rates were more frequent if the delivered dose was higher than 68 Gy (88). UK guidelines proposed that doses over 60 Gy may be employed. In patients with significant life-limiting comorbidities lower dose and palliative radiotherapy is an option (89).

\section{Treatment complications}

Multiple studies have characterized treatment toxicity, including nausea, vomiting, diarrhea, small bowel obstruction neuropathy, hydronephrosis and vaginal fistula, related to irradiation in patients with RPS. In a recent systematic review, Cheng et al. analyzed RT toxicity reported in the literature $(18.8 \% \mathrm{G} 1,10.2 \% \mathrm{G} 2,16.3 \% \mathrm{G} 3$, and $0.7 \%$ G4) (83). Among all toxicities, gastrointestinal (GI) toxicity is the best documented. Indeed, Gilbeau et al. described their experience with 45 patients treated with AdjRT (40-60 Gy). Three patients experienced 
grade 3-4 GI toxicity, and one patient died from intestinal bleeding (90). In a series of 79 patients treated with irradiation with curative or palliative intent, Catton et al. reported acute GI toxicity in $27 \%$ of patients (91). Zlotecki et al. published their experience with 40 patients treated with NeoRT and AdjRT irradiation. There was significantly more acute grade 1-2 enteritis in the AdjRT group compared to the NeoRT group ( $\mathrm{P}=0.0098)$ (66). Another study from Pezner et al. reported acute and late GI toxicity of AdjRT in 33 patients; approximately $79 \%$ and $10 \%$ of patients developed grade 1 to 2 and grade 3 to 4 acute GI toxicities, respectively (68). Moreover, $15 \%$ of patients developed late GI side effects. When NeoRT and AdjRT schedules were compared, Ballo et al. showed in their series that all patients who developed clinically significant radiation-related toxicities received AdjRT (64). Patients who receive NeoRT likely have a modestly increased risk of delayed wound healing, depending on the RT dosefractionation schedule. The acute toxicity of RT is related primarily to irradiating a large volume of the small bowel and is likely to be less pronounced with NeoRT compared to AdjRT because the tumor displaces much of the small bowel out of the RT field if the former is employed. NeoRT is most likely associated with a lower risk of small bowel injury because a smaller volume of the bowel is irradiated, and there are likely to be fewer adhesions at the time of treatment compared to AdjRT, allowing digestive tract motions, i.e., the delineated volumes are not necessarily those that are irradiated. In large resected tumors, digestive tract consequently fills the operative bed, and the volume of irradiated bowel track is dramatically increased compared to the bowel track compressed and tight against the tumor in the NeoRT schedule. Comparing to bowel bag constraints used for the gastrointestinal and gynecological cancers, Mak et al. evaluated dose constraints less stringent for NeoRT and showed that $\mathrm{V}_{30 \text { Gy }}$ was predictive of acute GI toxicity (92).

The kidneys are also dose-limiting organs for radiotherapy to upper abdominal cancers. Currently, data evaluating kidney injury after RPS irradiation is lacking (93). As aggressive surgery with nephrectomy is a part of the treatment for RPS, it is complicated to evaluate the potential effect of each treatment. Recently, a study from Massachusetts General Hospital evaluated the longterm effects of nephrectomy as a part of therapy in 54 patients with RPS. They showed that even though $56 \%$ of patients exhibited a worsening of their chronic kidney disease, no patient progressed to end-stage renal disease (94).
When treated with NeoRT, multidisciplinary management with surgery is necessary, as nephrectomy could be a part of the operation. If this is the case, the dose constraint to the contralateral kidney should be respected. If nephrectomy is not necessary, both organs should be spared, and it is essential to evaluate renal function carefully prior to RT.

Recently, Palm et al. presented the use of the Revised Edmonton Symptom Assessment Scale (ESAS-r-CSS) reported by patients during radiotherapy. The observed correlation between symptoms or side effects with covariate effects of age, gender, dose, tumor size, and location may offer the opportunity to minimize radiation effect by preventing them or managing them earlier (95).

\section{Prognostic factors and specific nomograms}

Histologic subtype appears to be an important predictor of prognosis. Compared to other histologic subtypes, the outcome is more favorable for well-differentiated LPSs (WDLPS), as it lacks metastatic potential. However, the risk of local recurrence persists for years after surgical resection. Leiomyosarcoma is more aggressive and has strong metastatic potential, with a 5 -year distant metastasis rate of $>50 \%$, but isolated LR is rare $(8,12)$. Finally, dedifferentiated LPS could relapse locally or distantly $(8,12,13,30)$. In a study performed by Lewis et al., patients with LPSs had a significantly lower local control rate compared to other patients (HR $=2.695 \%$ CI: 1.5-4.6; $\mathrm{P}=0.01$ ). In addition to histologic subtype, tumor size, grade, and completeness of surgical resection appear to influence LR risk (74). Indeed, a study performed by Stoeckle et al. revealed that incomplete resection $(\mathrm{P}=0.0005)$ and grade 3 histology $(\mathrm{P}=0.0017)$ adversely impacted survival (11). Gronchi et al. reported their experience with 167 patients showing that only histologic grade $(\mathrm{P}=0.0183)$ impacted OS (96). In another study, Singer et al. analyzed 83 patients who underwent surgery alone or combined with irradiation or chemotherapy between 1970 and 1994. Multivariate analysis revealed that intermediate-grade histology ( $\mathrm{P}=0.009)$, high-grade histology $(\mathrm{P}=0.008)$, and macroscopic residual disease $(\mathrm{P}=0.001)$ were associated with decreased OS (22).

Recently, Wong et al. analyzed prognostic factors used in the nomogram in their series. In multivariate analysis for OS and specific sarcoma survival, tumor size, and multifocality were prognosticators (97). For clinicians, evaluating patient prognosis is a key issue and is necessary for adapting treatment. In recent years, newer tools for 
prognosis prediction, such as nomograms, have been applied for STS. The group from Memorial Sloan Kettering Cancer Center (MSKCC) validated the first nomogram combining age at diagnosis, tumor size $(\mathrm{cm})$, grade, histological subtype, depth, and anatomical site, to predict 12-year sarcoma-specific survival (98). The nomogram computes two separate predictions: one for patients with low-grade tumors and the other for patients with highgrade tumors. A second nomogram was published and used worldwide. The Scaculator included age at diagnosis, tumor size $(\mathrm{cm})$, grade, histological subtype, multifocality, and extend of resection to determine 7-year OS. DFS was also available by considering tumor size, grade, histological subtype, and multifocality. Both predictions can be used for operating on patients (30). These nomograms have also been recognized by the most recent edition of the American Joint Committee on Cancer (AJCC) staging system as alternative methods for staging RPS (30,99-101). Furthermore, prognostic tools specific for RPS have been proven to have a better stratification ability and predicted prognosis better than MSKSN in their respective subsets of patients. Available nomograms specific to RPS are now numerous $(10,30,102,103)$. It is noteworthy that OS was the only outcome in two of four nomograms, whereas diseasefree survival and OS were predicted in one nomogram. Finally, one of the most recently published nomograms evaluated disease-specific death, LR, and distant metastasis endpoints (10). Today, Gronchi's nomograms have been made available as a smartphone/tablet app named "Sarculator", making it easy to use by clinicians as predictive tools in day-to-day clinical practice.

Some of the variability noted in the predicted outcomes of the various RPS nomograms are likely due to a combination of better understanding of this unique site and variation in surgical strategies over the different time intervals reflected in the individual studies. All four nomograms take into consideration tumor histology as a covariate, but the number of histological categories is different between nomograms. Indeed, it is notable that nomograms of Gronchi et al. (30) and Tan et al. (10) integrated seven subtypes. In contrast, Anaya et al. (102) adopted a more limited 3-subtype classification (WDLPS $v s$. dedifferentiated LPS $v s$. other), and Ardoino et al. used five pathology subtypes (103).

Tumor size was also considered in all nomograms. When used as a dichotomous variable, a tumor size higher than a specified cut-off is associated with a worse prognosis. Surprisingly, when used as a continuous variable, this trend is reversed for tumors size $>30 \mathrm{~cm}$ (30). One would argue that tumors reaching that size without symptomatology could demonstrate more indolent behavior. This argument was confirmed when tumor size was used as a categorical variable with three groups. Indeed, in the nomogram by Tan et al., higher dimensions are associated with a lower risk of distant metastasis compared to standard sizes (10).

Tumor grade is another well-established prognostic factor in patients with RPS (8). FNCLCC grading is the standard and was used in two nomograms $(30,103)$. However, the nomogram by Tan et al. distinguishes "lowgrade" and "high-grade" LMS (10), and in the nomogram of Anaya et al., grading was not selected as a prognostic variable (102).

External validation is a crucial component of the predictive capacity of nomograms (30,97,104-106). However, to date, only the nomograms by Gronchi et al. and the MSKCC have been externally validated $(30,106)$. Thus, for a patient with primary RPS, nomograms from Gronchi et al. can predict both OS and DFS (30). The nomograms from Tan et al. can accurately predict distant metastasis and local relapse risk. Wong et al. performed an external validation of the Sarculator (30) and the MSKCC nomogram (98), for a series of 109 patients. The authors concluded that the concordance indices of both the 7-year OS from the Sarculator and the 8-year specific sarcoma survival had good discriminative ability. The other time points had an acceptable discriminative ability, but their concordance was inferior, probably because of the patient characteristic differences between the reference and studied groups (97).

However, the use of RT was a predictive covariable of disease-specific survival and distant metastasis (10). Disappointingly, RT was not considered to predict LC in this nomogram. The last STRASS trial failed to demonstrate a benefit of NeoRT to overall sarcomas. However, the LPS subgroup could be improved thanks to NeoRT. Thus, to aid physicians, a specific LPS recurrence nomogram could be useful for indicating the relevance of RT.

Several considerations should be indicated for nomograms. Because treatments change over time, nomograms must be regularly upgraded and are at risk to become outdated (107). Furthermore, if nomograms predict patient outcome the results are not introduced in trials as a stratification variable to improve treatment. Their role remains eminently passive with a limited implication in the physician decision because the change of treatment 
management does not seem to change the prognostic. Indeed, only surgery was an influencing variable, but neither radiotherapy nor chemotherapy, whatever their schedule, neoadjuvant or adjuvant, were predictive.

\section{Conclusions and treatment guidelines}

Complete surgical resection of RPS remains the mainstay of treatment, as it provides the most significant impact on LC and survival. However, with complete resection alone, local relapse ranges from $45 \%$ to $86 \%$. NeoRT and AdjRT seem unable to improved OS although both decrease local relapses. Using advanced techniques of irradiation such as IG-IMRT or proton therapy, could increase the therapeutic ratio, by sparing OARs and permitting dose escalation to a high-risk target volume. The results of the EORTC randomized trials STRASS could be considered disappointing. The role of radiotherapy should be redesigned. Patients who may benefit from radiotherapy should be highly selected and managed in centers with a high level of expertise in sarcoma that use modern radiotherapy techniques. Moreover, close collaboration between radiation oncologists and surgeons is needed. Finally, using recent multi-institutional validated nomograms could help clinicians make decisions but creating nomograms to guide therapeutic decision could be more relevant than to predict patient outcome without therapeutic options to change it.

\section{Acknowledgments}

Funding: None.

\section{Footnote}

Reporting Checklist: The authors have completed the Narrative Review reporting checklist (available at http:// dx.doi.org/10.21037/cco-20-209).

Peer Review File: Available at http://dx.doi.org/10.21037/ cco-20-209

Conflicts of Interest: All authors have completed the ICMJE uniform disclosure form (available at http://dx.doi. org/10.21037/cco-20-209). The authors have no conflicts of interest to declare.

Ethical Statement: The authors are accountable for all aspects of the work in ensuring that questions related to the accuracy or integrity of any part of the work are appropriately investigated and resolved.

Open Access Statement: This is an Open Access article distributed in accordance with the Creative Commons Attribution-NonCommercial-NoDerivs 4.0 International License (CC BY-NC-ND 4.0), which permits the noncommercial replication and distribution of the article with the strict proviso that no changes or edits are made and the original work is properly cited (including links to both the formal publication through the relevant DOI and the license). See: https://creativecommons.org/licenses/by-nc-nd/4.0/.

\section{References}

1. Ducimetière F, Lurkin A, Ranchere-Vince $\mathrm{D}$, et al. Incidence of sarcoma histotypes and molecular subtypes in a prospective epidemiological study with central pathology review and molecular testing. PLoS One 2011;6:e20294.

2. ESMO/European Sarcoma Network Working Group. Soft tissue and visceral sarcomas: ESMO Clinical Practice Guidelines for diagnosis, treatment and follow-up. Ann Oncol 2014;25 Suppl 3:iii102-12.

3. Fletcher CDM, Bridge JA, Hogendoorn PCW, et al. WHO Classification of Tumours of Soft Tissue and Bone. [cited 2017 Aug 13]. Available online: http://publications. iarc.fr/Book-And-Report-Series/Who-Iarc-ClassificationOf-Tumours/Who-Classification-Of-Tumours-Of-SoftTissue-And-Bone-2013

4. Neuville A. Contribution of molecular biology for better management of soft tissue tumors. Ann Pathol 2012;32:S103-7.

5. Terrier P. Liposarcomas. Ann Pathol 2012;32:S108-10.

6. De Amorim Bernstein K, Delaney TF. Role of radiation therapy for non-extremity soft tissue sarcomas. J Surg Oncol 2015;111:604-14.

7. van Dalus T, van Geel AN, van Coevorden F, et al. Soft tissue carcinoma in the retroperitoneum: an often neglected diagnosis. Eur J Surg Oncol 2001;27:74-9.

8. Gronchi A, Strauss DC, Miceli R, et al. Variability in Patterns of Recurrence After Resection of Primary Retroperitoneal Sarcoma (RPS): A Report on 1007 Patients From the Multi-institutional Collaborative RPS Working Group. Ann Surg 2016;263:1002-9.

9. Mendenhall WM, Zlotecki RA, Hochwald SN, et al. Retroperitoneal soft tissue sarcoma. Cancer 2005;104:669-75. 10. Tan MC, Brennan MF, Kuk D, et al. Histology-based 
Classification Predicts Pattern of Recurrence and Improves Risk Stratification in Primary Retroperitoneal Sarcoma. Ann Surg 2016;263:593-600.

11. Stoeckle E, Coindre JM, Bonvalot S, et al. Prognostic factors in retroperitoneal sarcoma: a multivariate analysis of a series of 165 patients of the French Cancer Center Federation Sarcoma Group. Cancer 2001;92:359-68.

12. Toulmonde M, Bonvalot S, Meeus P, et al. Retroperitoneal sarcomas: patterns of care at diagnosis, prognostic factors and focus on main histological subtypes: a multicenter analysis of the French Sarcoma Group. Ann Oncol 2014;25:735-42.

13. Lahat G, Anaya DA, Wang X, et al. Resectable welldifferentiated versus dedifferentiated liposarcomas: two different diseases possibly requiring different treatment approaches. Ann Surg Oncol 2008;15:1585-93.

14. Manaster BJ. Soft-tissue masses: optimal imaging protocol and reporting. AJR Am J Roentgenol 2013;201:505-14.

15. Baheti AD, O'Malley RB, Kim S, et al. Soft-Tissue Sarcomas: An Update for Radiologists Based on the Revised 2013 World Health Organization Classification. AJR Am J Roentgenol 2016;206:924-32.

16. Shiraev T, Pasricha SS, Choong P, et al. Retroperitoneal sarcomas: a review of disease spectrum, radiological features, characterisation and management. J Med Imaging Radiat Oncol 2013;57:687-700.

17. Nanni C, Marzola MC, Rubello D, et al. Positron emission tomography for the evaluation of soft-tissue sarcomas and bone sarcomas. Eur J Nucl Med Mol Imaging 2009;36:1940-3.

18. Alford S, Choong P, Chander S, et al. Value of PET scan in patients with retroperitoneal sarcoma treated with preoperative radiotherapy. Eur J Surg Oncol 2012;38:176-80.

19. Fuglø HM, Jorgensen SM, Loft A, et al. The diagnostic and prognostic value of (1)(8)F-FDG PET/CT in the initial assessment of high-grade bone and soft tissue sarcoma. A retrospective study of 89 patients. Eur J Nucl Med Mol Imaging 2012;39:1416-24.

20. Kasraeian S, Allison DC, Ahlmann ER, et al. A comparison of fine-needle aspiration, core biopsy, and surgical biopsy in the diagnosis of extremity soft tissue masses. Clin Orthop Relat Res 2010;468:2992-3002.

21. Dei Tos AP. Liposarcomas: diagnostic pitfalls and new insights. Histopathology 2014;64:38-52.

22. Singer S, Antonescu CR, Riedel E, et al. Histologic subtype and margin of resection predict pattern of recurrence and survival for retroperitoneal liposarcoma. Ann Surg
2003;238:358-70; discussion 370-1.

23. Gutierrez JC, Perez EA, Franceschi D, et al. Outcomes for soft-tissue sarcoma in 8249 cases from a large state cancer registry. J Surg Res 2007;141:105-14.

24. Bonvalot S, Rivoire M, Castaing M, et al. Primary retroperitoneal sarcomas: a multivariate analysis of surgical factors associated with local control. J Clin Oncol 2009;27:31-7.

25. Gutierrez JC, Perez EA, Moffat FL, et al. Should soft tissue sarcomas be treated at high-volume centers? An analysis of 4205 patients. Ann Surg 2007;245:952-8.

26. Keung EZ, Chiang YJ, Cormier JN, et al. Treatment at low-volume hospitals is associated with reduced short-term and long-term outcomes for patients with retroperitoneal sarcoma. Cancer 2018;124:4495-4503.

27. van Dalen T, Hennipman A, Van Coevorden F, et al. Evaluation of a clinically applicable post-surgical classification system for primary retroperitoneal soft-tissue sarcoma. Ann Surg Oncol 2004;11:483-90.

28. Stahl JM, Corso CD, Park HS, et al. The effect of microscopic margin status on survival in adult retroperitoneal soft tissue sarcomas. Eur J Surg Oncol 2017;43:168-74.

29. Peacock O, Patel S, Simpson JA, et al. A systematic review of population-based studies examining outcomes in primary retroperitoneal sarcoma surgery. Surg Oncol 2019;29:53-63.

30. Gronchi A, Miceli R, Shurell E, et al. Outcome prediction in primary resected retroperitoneal soft tissue sarcoma: histology-specific overall survival and disease-free survival nomograms built on major sarcoma center data sets. J Clin Oncol 2013;31:1649-55.

31. Ray-Coquard I, Montesco MC, Coindre JM, et al. Sarcoma: concordance between initial diagnosis and centralized expert review in a population-based study within three European regions. Ann Oncol 2012;23:2442-9.

32. Almond LM, Gronchi A, Strauss D, et al. Neoadjuvant and adjuvant strategies in retroperitoneal sarcoma. Eur J Surg Oncol 2018;44:571-9.

33. Miura JT, Charlson J, Gamblin TC, et al. Impact of chemotherapy on survival in surgically resected retroperitoneal sarcoma. Eur J Surg Oncol 2015;41:1386-92.

34. Strauss DC, Hayes AJ, Thomas JM. Retroperitoneal tumours: review of management. Ann R Coll Surg Engl 2011;93:275-80.

35. Bonvalot S, Gronchi A, Le Pechoux C, et al. STRASS (EORTC 62092): A phase III randomized study of 
preoperative radiotherapy plus surgery versus surgery alone for patients with retroperitoneal sarcoma. J Clin Oncol 2019;37:11001.

36. Gronchi A, Miceli R, Allard MA, et al. Personalizing the approach to retroperitoneal soft tissue sarcoma: histologyspecific patterns of failure and postrelapse outcome after primary extended resection. Ann Surg Oncol 2015;22:1447-54.

37. Van De Voorde L, Delrue L, van Eijkeren M, et al. Radiotherapy and surgery-an indispensable duo in the treatment of retroperitoneal sarcoma. Cancer 2011;117:4355-64.

38. Trans-Atlantic RPS Working Group. Management of Primary Retroperitoneal Sarcoma (RPS) in the Adult: A Consensus Approach From the Trans-Atlantic RPS Working Group. Ann Surg Oncol 2015;22:256-63.

39. von Mehren M, Randall RL, Benjamin RS, et al. Soft Tissue Sarcoma, Version 2.2018, NCCN Clinical Practice Guidelines in Oncology. J Natl Compr Canc Netw 2018;16:536-63.

40. Haas RLM, Bonvalot S, Miceli R, et al. Radiotherapy for retroperitoneal liposarcoma: A report from the Transatlantic Retroperitoneal Sarcoma Working Group. Cancer 2019;125:1290-300.

41. Bremjit PJ, Jones RL, Chai X, et al. A contemporary large single-institution evaluation of resected retroperitoneal sarcoma. Ann Surg Oncol 2014;21:2150-8.

42. Chouliaras K, Senehi R, Ethun CG, et al. Role of radiation therapy for retroperitoneal sarcomas: An eight-institution study from the US Sarcoma Collaborative. J Surg Oncol 2019;120:1227-34.

43. Nussbaum DP, Rushing CN, Lane WO, et al. Preoperative or postoperative radiotherapy versus surgery alone for retroperitoneal sarcoma: a case-control, propensity scorematched analysis of a nationwide clinical oncology database. Lancet Oncol 2016;17:966-75.

44. Albertsmeier M, Rauch A, Roeder F, et al. External Beam Radiation Therapy for Resectable Soft Tissue Sarcoma: A Systematic Review and Meta-Analysis. Ann Surg Oncol 2018;25:754-67.

45. Diamantis A, Baloyiannis I, Magouliotis DE, et al. Perioperative radiotherapy versus surgery alone for retroperitoneal sarcomas: a systematic review and metaanalysis. Radiol Oncol 2020;54:14-21.

46. Caudle AS, Tepper JE, Calvo BF, et al. Complications associated with neoadjuvant radiotherapy in the multidisciplinary treatment of retroperitoneal sarcomas. Ann Surg Oncol 2007;14:577-82.
47. Tzeng CW, Fiveash JB, Popple RA, et al. Preoperative radiation therapy with selective dose escalation to the margin at risk for retroperitoneal sarcoma. Cancer 2006;107:371-9.

48. Baldini EH, Abrams RA, Bosch W, et al. Retroperitoneal Sarcoma Target Volume and Organ at Risk Contour Delineation Agreement Among NRG Sarcoma Radiation Oncologists. Int J Radiat Oncol Biol Phys 2015;92:1053-9.

49. Baldini EH, Wang D, Haas RL, et al. Treatment Guidelines for Preoperative Radiation Therapy for Retroperitoneal Sarcoma: Preliminary Consensus of an International Expert Panel. Int J Radiat Oncol Biol Phys 2015;92:602-12.

50. Wong P, Dickie C, Lee D, et al. Spatial and volumetric changes of retroperitoneal sarcomas during pre-operative radiotherapy. Radiother Oncol 2014;112:308-13.

51. Song T, Shen J, Liang BL, et al. Retroperitoneal liposarcoma: MR characteristics and pathological correlative analysis. Abdom Imaging 2007;32:668-74.

52. Sanguineti G, Little M, Endres EJ, et al. Comparison of three strategies to delineate the bowel for whole pelvis IMRT of prostate cancer. Radiother Oncol 2008;88:95-101.

53. Jabbour SK, Hashem SA, Bosch W, et al. Upper abdominal normal organ contouring guidelines and atlas: a Radiation Therapy Oncology Group consensus. Pract Radiat Oncol 2014;4:82-9.

54. McBride SM, Raut CP, Lapidus M, et al. Locoregional recurrence after preoperative radiation therapy for retroperitoneal sarcoma: adverse impact of multifocal disease and potential implications of dose escalation. Ann Surg Oncol 2013;20:2140-7.

55. Pisters PW, Ballo MT, Fenstermacher MJ, et al. Phase I trial of preoperative concurrent doxorubicin and radiation therapy, surgical resection, and intraoperative electronbeam radiation therapy for patients with localized retroperitoneal sarcoma. J Clin Oncol 2003;21:3092-7.

56. Swanson EL, Indelicato DJ, Louis D, et al. Comparison of three-dimensional (3D) conformal proton radiotherapy (RT), 3D conformal photon RT, and intensity-modulated RT for retroperitoneal and intra-abdominal sarcomas. Int J Radiat Oncol Biol Phys 2012;83:1549-57.

57. Yoon SS, Chen YL, Kirsch DG, et al. Proton-beam, intensity-modulated, and/or intraoperative electron radiation therapy combined with aggressive anterior surgical resection for retroperitoneal sarcomas. Ann Surg Oncol 2010;17:1515-29.

58. Baldini EH, Bosch W, Kane JM 3rd, et al. Retroperitoneal sarcoma (RPS) high risk gross tumor volume boost (HR GTV boost) contour delineation agreement among NRG 
sarcoma radiation and surgical oncologists. Ann Surg Oncol 2015;22:2846-52.

59. Database C. T. EORTC. [cited 2018 Mar 8]. Available online: http://www.eortc.org/research_field/clinicaldetail/62092\&T/

60. Bonvalot S, Gronchi A, Le Pechoux C, et al. Preoperative radiotherapy plus surgery versus surgery alone for patients with primary retroperitoneal sarcoma (EORTC-62092: STRASS): a multicentre, open-label, randomised, phase 3 trial. Lancet Oncol 2020;21:1366-77.

61. Bishop AJ, Zagars GK, Torres KE, et al. Combined Modality Management of Retroperitoneal Sarcomas: A Single-Institution Series of 121 Patients. Int J Radiat Oncol Biol Phys 2015;93:158-65.

62. Bossi A, De Wever I, Van Limbergen E, et al. Intensity modulated radiation-therapy for preoperative posterior abdominal wall irradiation of retroperitoneal liposarcomas. Int J Radiat Oncol Biol Phys 2007;67:164-70.

63. DeLaney TF, Chen YL, Baldini EH, et al. Phase 1 trial of preoperative image guided intensity modulated proton radiation therapy with simultaneously integrated boost to the high risk margin for retroperitoneal sarcomas. Adv Radiat Oncol 2017;2:85-93.

64. Ballo MT, Zagars GK, Pollock RE, et al. Retroperitoneal soft tissue sarcoma: an analysis of radiation and surgical treatment. Int J Radiat Oncol Biol Phys 2007;67:158-63.

65. Le Péchoux C, Musat E, Baey C, et al. Should adjuvant radiotherapy be administered in addition to frontline aggressive surgery (FAS) in patients with primary retroperitoneal sarcoma? Ann Oncol 2013;24:832-7.

66. Zlotecki RA, Katz TS, Morris CG, et al. Adjuvant radiation therapy for resectable retroperitoneal soft tissue sarcoma: the University of Florida experience. Am J Clin Oncol 2005;28:310-6.

67. Koshy M, Landry JC, Lawson JD, et al. Intensity modulated radiation therapy for retroperitoneal sarcoma: a case for dose escalation and organ at risk toxicity reduction. Sarcoma 2003;7:137-48.

68. Pezner RD, Liu A, Han C, et al. Dosimetric comparison of helical tomotherapy treatment and step-and-shoot intensity-modulated radiotherapy of retroperitoneal sarcoma. Radiother Oncol 2006;81:81-7.

69. Sargos P, Dejean C, de Figueiredo BH, et al. Highdose pre-operative helical tomotherapy (54 Gy) for retroperitoneal liposarcoma. Radiat Oncol 2012;7:214.

70. Woodward WA, Amos RA. Proton Radiation Biology Considerations for Radiation Oncologists. Int J Radiat Oncol Biol Phys 2016;95:59-61.
71. Serizawa I, Kagei K, Kamada T, et al. Carbon ion radiotherapy for unresectable retroperitoneal sarcomas. Int J Radiat Oncol Biol Phys 2009;75:1105-10.

72. Abu-Hijlih R, Mheid S, Abuhijla F, et al. Adaptive radiotherapy in patients receiving neoadjuvant radiation for soft tissue sarcoma. Rep Pract Oncol Radiother 2019;24:263-8.

73. Molina G, Hull MA, Chen YL, et al. Preoperative radiation therapy combined with radical surgical resection is associated with a lower rate of local recurrence when treating unifocal, primary retroperitoneal liposarcoma. J Surg Oncol 2016;114:814-20.

74. Lewis JJ, Leung D, Woodruff JM, et al. Retroperitoneal soft-tissue sarcoma: analysis of 500 patients treated and followed at a single institution. Ann Surg 1998;228:355-65.

75. Toulmonde M, Bonvalot S, Ray-Coquard I, et al. Retroperitoneal sarcomas: patterns of care in advanced stages, prognostic factors and focus on main histological subtypes: a multicenter analysis of the French Sarcoma Group. Ann Oncol 2014;25:730-4.

76. Sindelar WF, Kinsella TJ, Chen PW, et al. Intraoperative radiotherapy in retroperitoneal sarcomas. Final results of a prospective, randomized, clinical trial. Arch Surg 1993;128:402-10.

77. Kirste S, Landenberger N, Scholber J, et al. Retroperitoneal soft tissue sarcoma: low-dose neoadjuvant radiation therapy followed by surgery with or without intraoperative radiotherapy and adjuvant radiation therapy. Strahlenther Onkol 2019;195:558-65.

78. Gieschen HL, Spiro IJ, Suit HD, et al. Long-term results of intraoperative electron beam radiotherapy for primary and recurrent retroperitoneal soft tissue sarcoma. Int J Radiat Oncol Biol Phys 2001;50:127-31.

79. Sampath S, Hitchcock YJ, Shrieve DC, et al. Radiotherapy and extent of surgical resection in retroperitoneal softtissue sarcoma: multi-institutional analysis of 261 patients. J Surg Oncol 2010;101:345-50.

80. Turner BT, Hampton L, Schiller D, et al. Neoadjuvant radiotherapy followed by surgery compared with surgery alone in the treatment of retroperitoneal sarcoma: a population-based comparison. Curr Oncol 2019;26:e766-72.

81. Kelly KJ, Yoon SS, Kuk D, et al. Comparison of Perioperative Radiation Therapy and Surgery Versus Surgery Alone in 204 Patients With Primary Retroperitoneal Sarcoma: A Retrospective 2-Institution Study. Ann Surg 2015;262:156-62.

82. Snow HA, Hitchen TX, Head J, et al. Treatment of patients with primary retroperitoneal sarcoma: predictors 
of outcome from an Australian specialist sarcoma centre. ANZ J Surg 2018;88:1151-7.

83. Cheng H, Miura JT, Lalehzari M, et al. Neoadjuvant radiotherapy for retroperitoneal sarcoma: A systematic review. J Surg Oncol 2016;113:628-34.

84. Trovik LH, Ovrebo K, Almquist M, et al. Adjuvant radiotherapy in retroperitoneal sarcomas. A Scandinavian Sarcoma Group study of 97 patients. Acta Oncol 2014;53:1165-72.

85. Ecker BL, Peters MG, McMillan MT, et al. Preoperative radiotherapy in the management of retroperitoneal liposarcoma. Br J Surg 2016;103:1839-46.

86. Bates JE, Dhakal S, Mazloom A, et al. The Benefit of Adjuvant Radiotherapy in High-grade Nonmetastatic Retroperitoneal Soft Tissue Sarcoma: A SEER Analysis. Am J Clin Oncol 2018;41:274-9.

87. Wong P, Kassam Z, Springer AN, et al. Long-Term Quality of Life of Retroperitoneal Sarcoma Patients Treated with Pre-Operative Radiotherapy and Surgery. Cureus 2017;9:e1764.

88. Kepka L, DeLaney TF, Suit HD, et al. Results of radiation therapy for unresected soft-tissue sarcomas. Int J Radiat Oncol Biol Phys 2005;63:852-9.

89. Dangoor A, Seddon B, Gerrand C, et al. UK guidelines for the management of soft tissue sarcomas. Clin Sarcoma Res 2016;6:20.

90. Gilbeau L, Kantor G, Stoeckle E, et al. Surgical resection and radiotherapy for primary retroperitoneal soft tissue sarcoma. Radiother Oncol 2002;65:137-43.

91. Catton CN, O'Sullivan B, Kotwall C, et al. Outcome and prognosis in retroperitoneal soft tissue sarcoma. Int J Radiat Oncol Biol Phys 1994;29:1005-10.

92. Mak KS, Phillips JG, Barysauskas CM, et al. Acute gastrointestinal toxicity and bowel bag dose-volume parameters for preoperative radiation therapy for retroperitoneal sarcoma. Pract Radiat Oncol 2016;6:360-6.

93. Dawson LA, Kavanagh BD, Paulino AC, et al. Radiationassociated kidney injury. Int J Radiat Oncol Biol Phys 2010;76:S108-15.

94. Hull MA, Niemierko A, Haynes AB, et al. Post-operative renal function following nephrectomy as part of en bloc resection of retroperitoneal sarcoma (RPS). J Surg Oncol 2015;112:98-102.

95. Palm RF, Jim HSL, Boulware D, et al. Using the revised Edmonton symptom assessment scale during neoadjuvant radiotherapy for retroperitoneal sarcoma. Clin Transl Radiat Oncol 2020;22:22-8.

96. Gronchi A, Casali PG, Fiore M, et al. Retroperitoneal soft tissue sarcomas: patterns of recurrence in 167 patients treated at a single institution. Cancer 2004;100:2448-55.

97. Wong RX, Koh YS, Ong F, et al. Applicability of the Sarculator and MSKCC nomograms to retroperitoneal sarcoma prognostication in an Asian tertiary centre. Asian J Surg 2020;43:1078-85.

98. Kattan MW, Leung DH, Brennan MF. Postoperative nomogram for 12-year sarcoma-specific death. J Clin Oncol 2002;20:791-6.

99. van Houdt WJ, Raut CP, Bonvalot $S$, et al. New research strategies in retroperitoneal sarcoma. The case of TARPSWG, STRASS and RESAR: making progress through collaboration. Curr Opin Oncol 2019;31:310-6.

100. Chou YS, Liu CY, Chang YH, et al. Prognostic factors of primary resected retroperitoneal soft tissue sarcoma: Analysis from a single asian tertiary center and external validation of gronchi's nomogram. J Surg Oncol 2016;113:355-60.

101. Raut CP, Callegaro D, Miceli R, et al. Predicting Survival in Patients Undergoing Resection for Locally Recurrent Retroperitoneal Sarcoma: A Study and Novel Nomogram from TARPSWG. Clin Cancer Res 2019;25:2664-71.

102. Anaya DA, Lahat G, Wang X, et al. Postoperative nomogram for survival of patients with retroperitoneal sarcoma treated with curative intent. Ann Oncol 2010;21:397-402.

103. Ardoino I, Miceli R, Berselli M, et al. Histology-specific nomogram for primary retroperitoneal soft tissue sarcoma. Cancer 2010;116:2429-36.

104. Eilber FC, Brennan MF, Eilber FR, et al. Validation of the postoperative nomogram for 12-year sarcoma-specific mortality. Cancer 2004;101:2270-5.

105.Mariani L, Miceli R, Kattan MW, et al. Validation and adaptation of a nomogram for predicting the survival of patients with extremity soft tissue sarcoma using a threegrade system. Cancer 2005;103:402-8.

106. Raut CP, Miceli R, Strauss DC, et al. External validation of a multi-institutional retroperitoneal sarcoma nomogram. Cancer 2016;122:1417-24.

107. Tattersall HL, Callegaro D, Ford SJ, et al. Staging, nomograms and other predictive tools in retroperitoneal soft tissue sarcoma. Chin Clin Oncol 2018;7:36.

Cite this article as: Le Fevre C, Waissi W, Chambrelant I, Noel G, Antoni D. A critical narrative review of radiotherapy for retroperitoneal soft tissue sarcoma. Chin Clin Oncol 2020;9(6):79. doi: 10.21037/сco-20-209 